PAPER PRESENTED AT 28TH EU PVSEC, PARIS, FRANCE, 2013

\title{
Electrons and holes in solar cells with partial rear contacts
}

\author{
Andres Cuevas* \\ Research School of Engineering, The Australian National University, Canberra ACT 0200, Australia
}

\begin{abstract}
When the metal contact of a silicon solar cell is restricted to a fraction of the rear surface, the flow of electrons and holes towards that contact is constricted, which is beneficial for minority charge carriers but detrimental for majority carriers. It is possible to describe their 2D/3D transport and determine their concentration in the vertical and transversal dimensions of the solar cell by separately studying the central region near the contact and the peripheral region surrounding it. A virtue of such geometric approach is that it establishes a link between analytical models and computer simulations, providing both physical insight and sufficient accuracy to optimise partial rear contact devices. In this paper, we extend a previous version of the geometric model to solar cells having a full-area, locally contacted dopant diffusion on the rear surface. The case for n-type versus p-type wafers is considered, point contacts are compared with line contacts, including the impact of the metal/ semiconductor resistance and bulk recombination is evaluated. Copyright (C) 2013 John Wiley \& Sons, Ltd.
\end{abstract}

\section{KEYWORDS}

solar cells; modelling; partial rear contacts; silicon

\author{
*Correspondence \\ Andres Cuevas, Research School of Engineering, The Australian National University, Canberra ACT 0200, Australia. \\ E-mail: Andres.Cuevas@anu.edu.au
}

Received 27 June 2013; Revised 20 August 2013; Accepted 18 September 2013

\section{INTRODUCTION}

In a solar cell, photons are absorbed in a semiconductor material, transferring their energy to electrons and holes. The electrochemical energy thus established in the semiconductor increases with the concentration of electrons and holes, but so does the rate at which they recombine. Making recombination as slow as possible is a fundamental premise of solar cell design. To transfer the electrochemical energy to an external circuit in the form of an electric current, the solar cell needs to have two selective contact regions, one for each kind of charge carrier. A selective hole transport layer should present not only a high conductivity for holes but also a poor conductivity for electrons. Thus blocked in one direction, electrons will preferentially flow towards the metal contact placed on the electron transport layer. The latter should have a low conductivity for holes, so that they will flow preferentially towards the hole transport layer. As a consequence of these two layers of preferential conductivity electrons and holes flow in opposite directions towards an external circuit, if one is connected to the solar cell.

In the most common implementation of silicon solar cells, the electron and hole transport layers are formed by diffusing dopants into the silicon wafer, forming $n^{+}$and $p^{+}$regions. These regions cannot block minority carriers completely, even when optimised. The main reasons are that Auger recombination in highly doped silicon is very strong and that recombination at the contact between the metal contact and the $n^{+}$or $p^{+}$semiconductor is difficult to avoid with conventional technology. Such metal contact can, nevertheless, be restricted to a small fraction of the device area, permitting to passivate most of the surface. This is indeed indispensable at the front, so that light can get through, but it can also be done at the back, for example in a bifacial $n^{+} p p^{+}$or $p^{+} n n^{+}$silicon solar cell structure. Electrons in the $n^{+}$electron transport layer and holes in the $p^{+}$hole transport layer will then need to flow laterally in order to reach the external metallic terminals. Such lateral transport requires that some of the light-generated electrochemical energy is spent as heat, a process frequently described by means of a series resistance. The $n^{+}$ and $p^{+}$diffusions themselves can be localised, thus reducing Auger recombination, in an approach exemplarily demonstrated by the back point contact solar cell [1]. In a more evolutionary approach, it is frequent to keep a uniform diffusion on the front side and create a localised diffusion on the back side or simply a localised metal contact. This paper focuses on this second class of devices, which can be generically called partial rear contact (PRC) solar cells, 
including those that have a localised $p^{+}$or $n^{+}$region at the rear and those where the metal makes contact to the base region directly.

There are several possibilities to model partial rear contact solar cells. 2D and 3D numerical simulation programmes such as Sentaurus Device [2], PC2D[3], CoBo [4], or Quokka [5] are comprehensive, but they can be obscure. Analytical models [6-11] provide intuitive understanding but have limited applicability. Most of them derive from the work by Fisher [6], who showed that in an ideal PRC device (negligible bulk recombination) there is a parallelism between minority and majority carriers as they flow towards the localised contact, the former to recombine and the latter to exit the solar cell. He described minority carrier recombination at the rear by means of an effective surface recombination velocity, mathematically linked to the same series resistance that impedes the flow of majority carriers. Once determined, this effective surface recombination velocity is inserted in 1D device models in order to determine the electrical parameters of the solar cell. Although such approach can be acceptable for well-designed PRC devices, it is hardly general. In particular, reducing the analysis to one dimension can make one lose sight of the true 2D/3D nature of the device.

The two-dimensional structure of PRC devices calls for special attention on the lateral transport of charge carriers. As we shall see in Section 3, the build-up of carrier concentration in the lateral direction leads to increased recombination losses. But the key consequence of a localised rear contact is that the flow of charge carriers, both majority and minority, is constricted, similarly to the flow of water in a funnel. For minority carriers, such constriction is equivalent to reducing the conductance in the direction of the rear contact, which helps to build up their concentration inside the solar cell. For majority carriers, a lower conductance means higher resistive losses. Hence, a trade-off needs to be established; and for that, a $2 \mathrm{D}$ or $3 \mathrm{D}$ physical model of carrier transport and recombination is needed. A relatively simple model based on a regional partition of the device has been developed recently, commencing with an analytical derivation of the open-circuit voltage $V_{\mathrm{oc}}$ and the series resistance $R_{s}$ in low injection conditions [11], and extending it to a full iterative device model in arbitrary injection for both circular [12] or linear rear contacts[13]. This geometric model was found to be in good agreement with $2 \mathrm{D}$ and $3 \mathrm{D}$ numerical simulation programmes for the case of low bulk and rear surface recombination [14]. In this paper, we extend the model further to devices that have a highly doped $p^{+}$or $n^{+}$conductive layer on the back surface, as well as to PRC cells made with n-type silicon to assess what advantages, if any, n-Si may offer compared with $\mathrm{p}$-Si. Although the mathematical expressions already derived for $\mathrm{p}-\mathrm{Si}$ can easily be extrapolated, we reproduce them here for n-Si as way of summarising the geometric model. We also explore its applicability to cases where recombination in the bulk is significant.

\section{KEY CARRIER TRANSPORT PHENOMENA IN THE CENTRAL, NEAR-CONTACT REGION}

Constricting charge carrier flow towards the rear contact has two primary beneficial effects on minority carriers and two ancillary detrimental effects on majority carriers. The first benefit, a reduction of the area where surface recombination is very strong, is counteracted by a potential increase in the contact resistance between metal and semiconductor. The second benefit is related to the reduced volume of semiconductor material available for current flow ('current crowding') in the base region, which results in a lower conductance for minority carriers towards the high recombination contact, but also in an increased resistance for majority carriers. Additional effects related to the interplay between lateral charge carrier transport and recombination will be discussed in the next section.

Let us focus first on PRC solar cells where the separation between rear contacts is relatively small, so that it is sufficient to describe then by means of what we shall call the central region of the device. As an example, Figure 1 shows the cross-sectional diagram, plotted to scale, for a $W=150 \mu \mathrm{m}$-thick PRC solar cell with rear point contacts of diameter $d=100 \mu \mathrm{m}$, separated $P=300 \mu \mathrm{m}$. The aperture area of the unit cell, having just one contact is $A_{O}=$ $P x 1 \mathrm{~cm}$ for a linear contact, $A_{O}=P^{2}$ for a square arrangement of circular point-like contacts or, for a hexagonal one,

$$
A_{o(\text { hex })}=\frac{\sqrt{3}}{2} P^{2}
$$

We consider in this section an n-type dopant density of $N_{D}=10^{16} \mathrm{~cm}^{-3}$, which ensures that low injection conditions prevail. For these, and the rest of calculations in the paper, we have assumed a good quality front surface region characterised by $J_{O f}=100 \mathrm{fA} \cdot \mathrm{cm}^{-2}$. This value is representative of experimental phosphorus and boron diffusions with a sheet resistance of about $R_{f}=100 \Omega / \mathrm{sq}$, a well-passivated front textured surface and a metal grid contact fraction of about 5\%, typical of industrial silicon solar cells. The photogenerated current density has been adjusted to $J_{\mathrm{ph}}=40 \mathrm{~mA} \cdot \mathrm{cm}^{-2}$, and shading losses due to

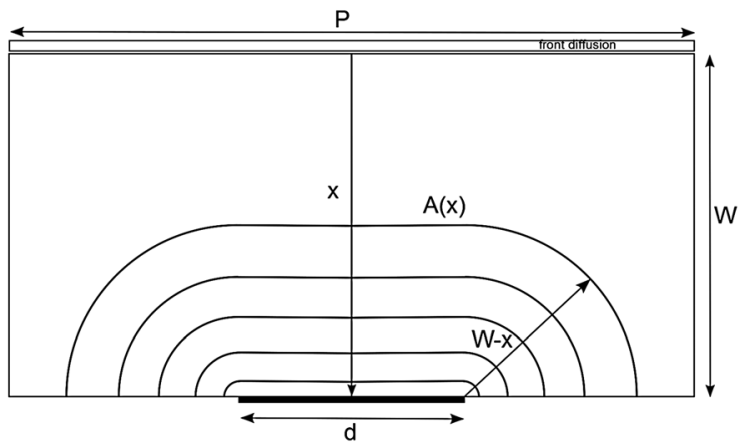

Figure 1. Cross-sectional diagram of the central region of a partial rear contact solar cell with thickness $W=150 \mu \mathrm{m}$, contact dimension $d=100 \mu \mathrm{m}$ and pitch $P=300 \mu \mathrm{m}$. 
the front metal grid have not been separately included. All the calculations have been made at $300 \mathrm{~K}$. We will start by neglecting all recombination except at the front surface and at the rear contact and by considering the simple case of open-circuit conditions.

\subsection{Benefits to minority carriers}

Recombination in the front $p^{+}$diffusion, labelled $J_{\text {rec }}^{f}$, can be expressed by means of a recombination current pre-factor $J_{O f}$, which can be interpreted as the thermal (equilibrium) recombination current density, as the following proportionality between recombination current and $p n$ product shows,

$$
\frac{J_{\mathrm{rec}}^{f}}{\left(n_{0}+p_{f}\right) p_{f}}=\frac{J_{0 f}}{n_{i}^{2}}
$$

where $p_{f}$ is the excess hole concentration at the front end of the base region, $q$ the elementary charge, $n_{i}$ the intrinsic carrier concentration and $n_{O} \approx N_{D}$ is the equilibrium electron concentration, approximately equal to the concentration of donors in the n-type base region. In open-circuit conditions, all the electrons and holes that survive recombination at the front surface will flow down towards the rear contact to recombine there. The hole and electron currents are equal and with opposite sign,

$$
I_{\mathrm{p}}(x)=-I_{\mathrm{n}}(x) \approx A_{0}\left(J_{\mathrm{ph}}-J_{\text {rec }}^{f}\right)
$$

where $J_{\mathrm{ph}}$ is the photogenerated current density. Note that while the hole current remains approximately constant in the vertical direction, the current density will be significantly higher at the rear contact. Recombination at the rear metal contact, including the localised $n^{+}$diffusion sometimes present underneath it, can be represented via a recombination current pre-factor $J_{O \text { cont }}$. In steady-state, the rate at which carriers recombine is equal to the rate at which they arrive to the surface

$$
A_{\text {cont }} J_{0 \text { cont }} \frac{\left(n_{0}+p_{\text {cont }}\right) p_{\text {cont }}}{n_{i}^{2}}=A_{0}\left(J_{\mathrm{ph}}-J_{\text {rec }}^{f}\right)
$$

The first important consequence of restricting the metal contact to a small fraction of the rear surface $f_{c}=A_{\text {cont }} / A_{O}$ is that, as shown by (4), the minority carrier concentration at the rear contact $p_{\text {cont }}$ increases proportionally, in order to speed up recombination at the contact to accommodate for the highly concentrated carrier flux reaching the surface. This intuitive result is illustrated in Figure 2, where the minority carrier concentration profile in the base region for a PRC cell with $f_{c}=10 \%$ is compared with a full-area contact $1 \mathrm{D}$ device for the case of $J_{O \text { cont }}=10^{-11} \mathrm{~A}$. $\mathrm{cm}^{-2}$, which in low injection equivalent to a surface recombination velocity $S_{\text {cont }}=6.6 \times 10^{3} \mathrm{~cm} / \mathrm{s}$. The respective carrier concentrations at the back surface are $p_{\text {cont }}$ (1D) $=3.41 \times 10^{13} \mathrm{~cm}^{-3}$ and $p_{\text {cont }(\mathrm{PRC})}=2.77 \times 10^{14} \mathrm{~cm}^{-3}$.

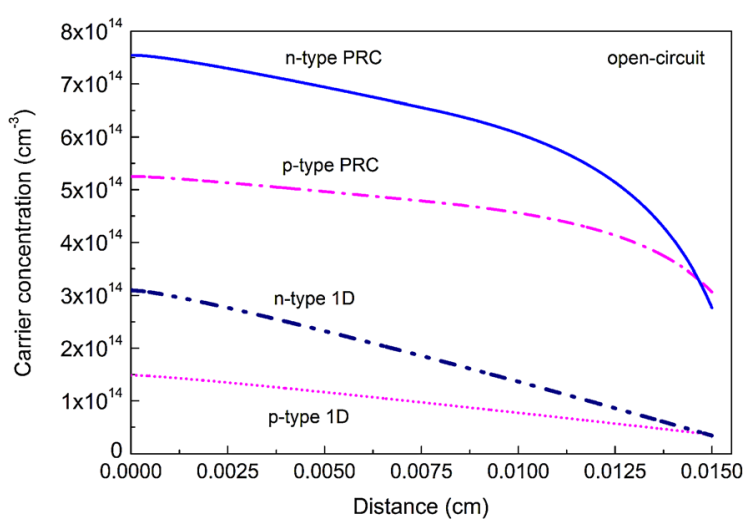

Figure 2. Carrier density profiles in open-circuit for $J_{\text {Ocont }}=$ $10 \mathrm{pA} \cdot \mathrm{cm}^{-2}\left(S_{\text {cont }}=6.6 \times 10^{3} \mathrm{~cm} \cdot \mathrm{s}^{-1}\right)$ partial rear contact point contact cell width $d=100 \mu \mathrm{m}$ and pitch $P=300 \mu \mathrm{m}$, compared with the case of a full-area contact. The wafer thickness is $W=150 \mu \mathrm{m}$ and the dopant density $N_{\text {dop }}=10^{16} \mathrm{~cm}^{-3}$, either n-type or p-type.

To understand the second beneficial effect, let us determine the minority carrier concentration at the front. The hole current $I_{p}(x)$ is driven by a gradient of the electrochemical potential for holes $-E_{F p}$ [15]. In low injection, the flow of minority carriers, holes in this case, can be expressed in terms of the gradient of their concentration

$$
I_{p}(x)=-\sigma_{p} A(x) \frac{1}{q} \frac{d E_{F p}}{d x} \approx-q D_{p} A(x) \frac{d p}{d x}
$$

where $\sigma_{p}$ is the hole conductivity, $D_{p}$ is the hole diffusion coefficient and $A(x)$ is the area crossed by the holes as they flow towards the rear contact. The following functions, drawn in Figure 1, are adequate to describe the crosssectional area as a function of the vertical position $x$ for linear contacts of width $d$ or for point contacts of diameter $d$, respectively [12],

$$
\begin{gathered}
A(x)_{\text {linear }}=[d+\pi(W-x)] 1 \mathrm{~cm} \\
A(x)_{p o \text { int }}=\pi(d / 2)^{2}+\pi^{2}(d / 2)(W-x)+2 \pi(W-x)^{2}
\end{gathered}
$$

The minority carrier concentration as a function of position can be obtained by integration of (5), together with (3) and (4),

$$
p(x)=p_{\text {cont }}+\frac{J_{\mathrm{ph}}-J_{\mathrm{rec}}^{f}}{q D_{p}} \int_{x}^{W} \frac{A_{0}}{A(x)} d x
$$

A consequence of constricting carrier flow inside a small cross-sectional area is that a stronger gradient of the minority carrier concentration is required to maintain a given current, compared with the 1D case, as illustrated in Figure 2. This is positive because it helps to slow down the flow of carriers towards the rear, hence the rate at which they recombine there. The detailed manner in which the cross-sectional area 
$A(x)$ varies with position affects the shape of the carrier density profile, but not very strongly, because the function $A(x)$ is integrated. In the example shown in Figures 1 and 2, $A(x)=A_{O}$ for approximately half of the wafer thickness, that is, the flow of carriers is 1D over that distance.

The combination of a higher minority carrier concentration at the rear contact and a constrained minority carrier transport in the semiconductor regions surrounding the contact leads to a globally higher carrier concentration and to a higher open-circuit voltage in the PRC solar cell $V_{\mathrm{oc}(\mathrm{PRC})}=649 \mathrm{mV}$ compared with the $1 \mathrm{D}$ case, where $V_{\mathrm{oc}(1 \mathrm{D})}=626 \mathrm{mV}$. The short-circuit current also improves thanks to the PRC design, leading to a conversion efficiency of $20.9 \%$, compared with $18.8 \%$ for the $1 \mathrm{D}$ case. If a p-type silicon wafer of the same doping were used, the voltages would be lower $V_{\mathrm{oc}(1 \mathrm{D})}=607 \mathrm{mV}$ and $V_{\mathrm{oc}(\mathrm{PRC})}=640 \mathrm{mV}$, due to the higher diffusivity of minority electrons, which means that the gradient of the carrier profile would be smaller for a given current, as shown in Figure 2.

\subsection{Detrimental impacts on majority carriers}

There are two types of majority carrier transport losses that mirror the two beneficial effects described previously. One is the resistance at the metal-semiconductor contact; the other is the resistance due to current crowding. The first detrimental consequence of having small rear contacts is that the contact resistance between the metal and the semiconductor increases in inverse proportion to the metal contact fraction,

$$
R_{s(\mathrm{cont})}=\frac{\rho_{c}}{f_{c}}
$$

where $\rho_{c}$ is the specific contact resistivity, expressed in $\Omega \mathrm{cm}^{2}$. Empirically, it has been observed that $\rho_{c}$ decreases with the dopant concentration at the surface of the semiconductor $[16,17]$. It can be expected to be very low for $\mathrm{p}^{+}$and $\mathrm{n}^{+}$diffused regions, but it can be high when the metal makes direct contact to the wafer. In the particular example discussed previously $f_{c}=10 \%$ is relatively high, and this makes the device relatively tolerant of high contact resistivities. For example, $\rho_{c}=10 \mathrm{~m} \Omega \mathrm{cm}^{2}$ produces a drop of just $4 \mathrm{mV}$ in the maximum power voltage.

To evaluate the crowding resistance, let us consider maximum power conditions. If we assume that electrons and holes are generated very close to the front surface, the net vertical current is approximately equal to the majority carrier current $I \approx I_{n}(x)$ over most of the wafer thickness. The latter is driven by a gradient of the electrochemical potential for electrons $E_{F n}[15]$

$$
I_{n}(x)=\frac{\sigma_{n} A(x)}{q} \frac{d E_{F n}}{d x}
$$

where $\sigma_{n}$ is the electron conductivity,

$$
\sigma_{n} \approx q \mu_{n}\left(N_{D}+p_{f}(x)\right)
$$

In low injection, the concentration of majority carriers is approximately uniform and $\sigma_{n}=q \mu_{n} N_{D}$. The electrochemical (almost exclusively electric) potential difference between the front and back surfaces can be found by integration,

$$
V(0)-V(W)=\frac{J_{n} A_{0}}{\sigma_{n}} \int_{W}^{0} \frac{1}{A(x)} d x
$$

An equivalent series resistance can be defined as the ratio between the electric potential difference and the current. Multiplying by the aperture area $A_{O}$, to express it in $\Omega \mathrm{cm}^{2}$,

$$
R_{s(\text { crowd })}=\frac{1}{\sigma_{n}} \int_{W}^{0} \frac{A_{0}}{A(x)} d x
$$

We can see that the same 'crowding' factor that helps to increase the minority carrier concentration according to (8) has the undesired effect of producing a resistive loss. The latter is, however, quite small in well-designed devices. For example, in the case of a $0.53 \Omega \mathrm{cm}$ n-type wafer with $\rho_{c}=0$, the equivalent resistance is $15 \mathrm{~m} \Omega \mathrm{cm}^{2}$, and the voltage drop is just $0.6 \mathrm{mV}$ in maximum power conditions.

Given that reducing the contact fraction to $10 \%$ leads to a significant increase in the output voltage that far outweighs the resistive losses, it is tempting to reduce the contact fraction even further. Let us expand the preceding device in the lateral dimension by increasing the pitch between the contacts to $P=0.1 \mathrm{~cm}$, which makes the metal contact fraction approximately equal to $1 \%$. Assuming that the excess hole concentration $p_{f}$ is uniform in the lateral direction, which is approximately true for this particular case, then we can still use (2) to determine the recombination loss at the front diffusion. An analytical solution for $V_{\mathrm{oc}}$ is then possible [11], and we can find that $V_{\mathrm{oc}(1 \%)}=676 \mathrm{mV}$ is significantly higher than $V_{\mathrm{oc}(10 \%)}=649 \mathrm{mV}$. The strongly concentrated minority carrier flux reaching the contact results in a minority carrier concentration $p_{\operatorname{cont}(1 \%)}=7.9 \times 10^{14} \mathrm{~cm}^{-3}$ that is 2.6 times higher than in the $f_{c}=10 \%$ case. The reason why they are not in a ratio of ten is because recombination losses at the front surface are higher in the case of $f_{c}=1 \%$. The crowding resistance is also higher, $147 \mathrm{~m} \Omega \mathrm{cm}^{2}$, and it produces a voltage drop of $5.6 \mathrm{mV}$. The main drawback is, however, that contact resistance becomes a serious problem; a $\rho_{c}=10 \mathrm{~m} \Omega \mathrm{cm}^{2}$ makes the efficiency fall from $22.6 \%$ to $21.2 \%$.

Although the expressions for the minority carrier concentration (8) and the series resistance (13) are useful to understand the basic mechanisms of PRC solar cells, similarly to previously reported analytical expressions [6-8], a more general analysis is necessary to account for position-dependent carrier recombination and for transport in high injection conditions. In general, the lateral transport of electrons and holes along the peripheral region, particularly when the pitch $P$ is large, can lead to additional losses than those considered until now. We discuss these matters in the next section. 


\section{REGIONAL ANALYSIS OF THE PARTIAL REAR CONTACT SOLAR CELL}

The simplest way to approach the PRC device in a generic way is by dividing it in two regions connected in parallel. The central region is defined by the sphere of influence of the local rear contact. It is there that the main effects of contact restriction and current crowding occur, as discussed previously. The rest of the solar cell, the peripheral region has a relatively passive role. Its function is to absorb photons and transfer the generated electrons and holes towards the central region. Our objective is to determine the net carrier current $I_{\text {lat }}$ that is eventually transferred.

\subsection{Recombination losses associated to the lateral transport of electrons and holes}

The lateral flow of electrons and holes requires the establishment of gradients in their respective electrochemical potentials, that is, gradients of the carrier concentrations themselves. If the distance is long or the conductivity is low, the concentration of carriers varies strongly as a function of the lateral position $y$. To evaluate more accurately the recombination losses in the peripheral region, we consider a number of relatively small elements and perform an iterative analysis. The process is described in detail in [13] for linear contacts; here, we apply it to circular contacts and extend it to the case in which an additional conductive layer is added to the rear side of the solar cell, as shown in Figure 3. For an n-type device, this $\mathrm{n}^{+}$layer would be a phosphorus diffusion. It is intuitive to regard the $n$-type base and the $n^{+}$layer as two conductances in parallel; the electron current will then be composed of the two contributions,

$$
I_{n}(y)=\frac{1}{q} \frac{d E_{F n}}{d y}\left(\sigma_{n} W+\frac{1}{R_{b}}\right) \cdot 2 \pi y
$$

where $R_{b}$ is the sheet resistance of the back $n^{+}$region. In an $\mathrm{n}$-type device, the front $\mathrm{p}^{+}$layer (a boron diffusion) is the primary conduit for the lateral transport of holes (holes may also flow through the base region, but we neglect such effect here),

$$
I_{p}(y)=\frac{1}{q R_{f}} \frac{d E_{F p}}{d y} \cdot 2 \pi y
$$

where $R_{f}$ is the sheet resistance of the front $p^{+}$region. Note that in writing (15), we have assumed a concentric circular path for the current in the front diffusion, that is, we have considered that the front metal contacts are also circular and placed exactly on top of the rear contacts. Although this is not usually the case in real solar cells with a front metal grid, it permits mathematical simplicity. The difference between the quasi-Fermi energies expressed in units of Volts as a function of the lateral position $V(y)$ can be obtained by integration of (14) and (15), noting that the electron and hole currents flowing out of a given elementary volume $d y$ are equal but with opposite sign. The voltage drop produced by the flow of current across a differential element $d y$ is

$$
d V(y)=I_{n}\left(\frac{1}{\sigma_{n} W+1 / R_{b}}+R_{f}\right) \frac{d y}{2 \pi y}
$$

$V(y)$ increases with distance, as a consequence of the resistive voltage drops in the semiconductor layers. Knowing the difference between the quasi-Fermi energies for electrons and holes, it is possible to determine the minority carrier concentration as a function of the lateral distance $p_{f}(y)$

$$
p_{f}(y)\left(n_{o}+p_{f}(y)\right)=n_{i}^{2} \exp \left(\frac{V(y)}{k T / q}\right)
$$

where $k$ is the Boltzmann constant and $T$ the temperature in degrees Kelvin. Because $V(y)$ increases with distance from the contact, so will the excess carrier concentration, triggering higher recombination losses. To determine the latter, we make the drastic simplification that at a given position $y$ the excess carrier density is approximately constant in the vertical dimension. The approximation works well when

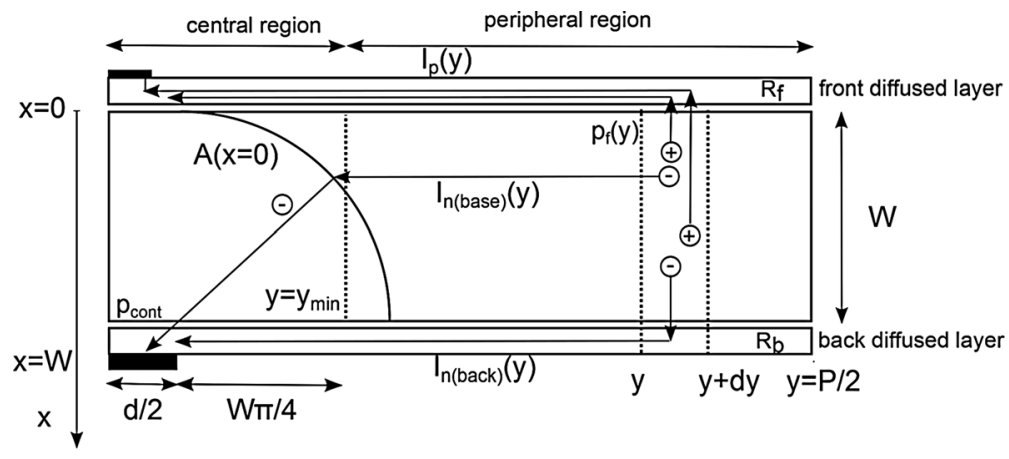

Figure 3. Cross-section of a half-unit element of partial rear contact solar cell showing the central and peripheral regions in which it is divided for the analysis. Drawn to scale for a wafer thickness $W=150 \mu \mathrm{m}$, rear contact dimension $d=100 \mu \mathrm{m}$, and pitch $P=1000 \mu \mathrm{m}$. The upper and lower layers (not to scale) represent $p^{+}$and $n^{+}$diffusions on this $n$-type solar cell. Two possible paths for electrons in the lateral region are illustrated, one through the base and the other through the rear diffusion. 
the minority carrier diffusion length is greater than the wafer thickness and the rear surface is well passivated. The recombination current as a function of the lateral position then is

$$
J_{\text {rec }}^{\text {lat }}(y) \approx\left(J_{0 f}+J_{0 \text { bpass }}\right) \frac{p_{f}(y)\left[n_{0}+p_{f}(y)\right]}{n_{i}^{2}}+q \frac{p_{f}(y) W}{\tau_{p}}
$$

where bulk recombination has been accounted for by means of an effective minority carrier lifetime $\tau_{p}$, which may represent a combination of defect-assisted and intrinsic recombination. The current continuity equation establishes the link between recombination, generation and transport

$$
I_{n}(y)=I_{n}(y+d y)+\left[J_{\mathrm{ph}}-J_{\text {rec }}^{\text {lat }}(y)\right] 2 \pi y d y
$$

Note that in the n-type device case, we are studying, electrons flow towards the left and $I_{n}$ is positive, therefore (19) indicates that the magnitude of $I_{n}$ increases towards the near-contact region, situated on the left. In the case of linear contacts, the product $2 \pi y$ in the aforementioned expressions should be replaced by $1 \mathrm{~cm}$.

To find a global solution for the complete device, it is necessary to follow an iterative procedure, changing the value of the minority carrier concentration at the farthest point of the unit cell $y_{\max }=P / 2$ until self-consistency is achieved. For a given value of $p_{f}(P / 2)$, the net current injected from the peripheral region into the near-contact region $I^{\text {at }}$ can be determined by subtracting from the total photogeneration occurring in the periphery the sum of all the contributions to recombination in it. Alternatively, $I^{\text {lat }}$ can be calculated as the value of the electron current at the boundary between the lateral and near-contact regions $I^{\text {lat }}=\left|I_{n}\left(y_{\min }\right)\right|$. In the geometric model of Figure 3 , the boundary is at $y_{\min }=d / 2+W \pi / 4$, with $d$ being the diameter of the point contact or the width of the line contact.

Figure 4 illustrates some carrier density profiles in the lateral region both in open-circuit and maximum power conditions. A strong carrier concentration gradient is required to drive carriers laterally over the relatively long distance modelled in this example, $P / 2=0.2 \mathrm{~cm}$. The gradient becomes less steep if an additional conductive layer of the same polarity is added at the rear surface. These carrier profiles are discussed in more detail in Section 4.

\subsection{Injection of carriers into the central region}

Once the lateral current arrives in the near-contact central region, it is subjected to the crowding effects described in Section 2. Nevertheless, it is necessary to exclude from crowding the fraction of current transported by the back conductive layer, when one is present (Figure 3). Such fraction $f_{\text {back }}$ is given by the ratio between the conductance of the rear diffusion to the total conductance presented by the peripheral region. We calculate the latter at the boundary between the lateral and central regions, that is, at $y=y_{\min }$,

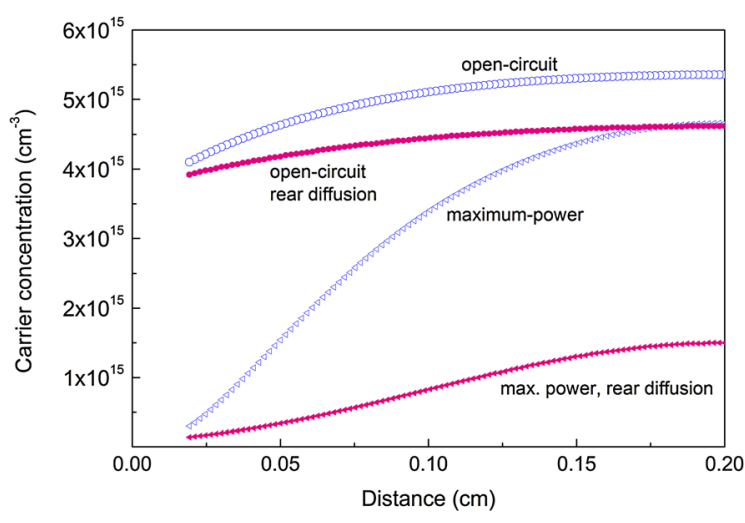

Figure 4. Minority carrier concentration as a function of the lateral distance from the centre of the contact. The case shown is $P=4 \mathrm{~mm}$, line contact width $d=150 \mu \mathrm{m}, J_{O f}=10^{-13} \mathrm{Acm}^{-2}$, $J_{\text {Ocont }}=1 \times 10^{-12} \mathrm{Acm}^{-2}$ and $N_{A}=10^{15} \mathrm{~cm}^{-3}$. With or without a $100 \mathrm{Ohm} / \mathrm{sq}$ rear diffusion with $J_{\text {Opass }}=30 \mathrm{fAcm}^{-2}$.

$$
\begin{aligned}
f_{\text {back }} & =\frac{I_{n(\text { back })}}{I_{n(\text { back })}+I_{n(\text { base })}} \\
& =\frac{1 / R_{b}}{\sigma_{n}+1 / R_{b}} \approx \frac{1 / R_{b}}{q \mu_{n}\left(N_{D}+p_{f}\left(y_{\text {min }}\right)\right)+1 / R_{b}}
\end{aligned}
$$

The vertical current subjected to crowding effects is related to the total device current by

$$
I^{\mathrm{vert}}=I-f_{\mathrm{back}} I^{\mathrm{lat}}
$$

The remainder of the peripheral current, that is, $\left(1-f_{\text {back }}\right)$ $I^{\text {lat }}$ is included in the analysis of the central region as a delta generation function at the front surface. This region can be modelled using semi-analytical expressions for the minority carrier concentration and the electrical potential that are valid for arbitrary injection conditions [18]. The most important expressions are summarised here for the case of an n-type device. The vertical current density $J^{\mathrm{vert}}$ is equal to the sum of the electron and hole currents:

$$
J^{\mathrm{vert}}=J_{\mathrm{n}}(x)+J_{\mathrm{p}}(x)
$$

An analysis based on quasi-neutrality permits to express the minority hole current as

$$
J_{\mathrm{p}}(x)=-q D_{\mathrm{eff}} \frac{A(x)}{A_{0}} \frac{d p}{d x}+J^{\mathrm{vert}} \frac{p D_{p}}{n D_{n}+p D_{p}}
$$

The effective diffusion coefficient $D_{\text {eff }}$ is

$$
D_{\mathrm{eff}}=\frac{(n+p) D_{n} D_{p}}{n D_{n}+p D_{p}}
$$

where $D_{n}$ and $D_{p}$ are the diffusion coefficients for electrons and holes. Integration of (22) permits to determine the minority carrier profile, in combination with the continuity 
equation (similar to (19) but in the vertical direction). The analysis also gives the electric field

$$
\xi=\frac{k T}{q} \frac{\mu_{p}-\mu_{n}}{n \mu_{n}+p \mu_{p}} \frac{d p}{d x}+\frac{J^{\mathrm{vert}} A_{0}}{q A(x)\left(n \mu_{n}+p \mu_{p}\right)}
$$

The second term is usually dominant, and it includes crowding effects (via the area ratio). The electrostatic potential drop $V_{\text {base }}$ is found from (25) by integration. It reduces the voltage between the upper and lower vertical contacts of the central region,

$$
V=\frac{k T}{q} \ln \left(\frac{\left(p_{o}+p_{f}\right)\left(n_{o}+p_{\text {cont }}\right)}{n_{i}^{2}}\right)+V_{\text {base }}
$$

It is possible to account for the additional, usually very small, voltage drop due to the flow of current through the back layer over the distance between $y=y_{\min }$, and the metal contact, $y=d / 2$. Such additional resistance is, for point or line contacts, respectively

$R_{b}^{\text {add }}$ (po int) $\left(\Omega c m^{2}\right)=f_{\text {back }} R_{b} \frac{A_{o}}{2 \pi}\left[\frac{y_{\text {min }}^{2}}{y_{\text {min }}^{2}-(d / 2)^{2}} \ln \left(\frac{y_{\text {min }}}{d / 2}\right)-\frac{1}{2}\right]$

$$
R_{b}^{\text {add }} \text { (line) }\left(\Omega \mathrm{cm}^{2}\right)=f_{\text {back }} R_{b} \frac{A_{o}}{4}\left(y_{\text {min }}-\frac{d}{2}\right)
$$

where the factor $f_{\text {back }}$ accounts for the fact that only a fraction of the total current flows through that additional resistance.

An advantage of the geometric approach as outlined in this section is that it can be easily implemented in computer simulation programmes, such as the Excel-based QsCell [19]. This permits to determine all the relevant device parameters, $J_{\mathrm{sc}}, V_{\mathrm{oc}}, F F$ and efficiency. In the following section, we apply it to discuss several cases of practical interest and physical significance.

\section{CASE STUDIES}

\subsection{The effects of a back surface conductive layer}

Adding a full-area conductive layer to the rear side of a PRC solar cell helps to transport carriers laterally from the peripheral region and avoid some of the losses that would otherwise occur. To increase the sensitivity to such losses, we select a wafer doping of $N_{\text {dop }}=10^{15} \mathrm{~cm}^{-3}$, either acceptors or donors, and a good quality front surface region with a thermal recombination current density $J_{O f}=100 \mathrm{fA} \cdot \mathrm{cm}^{-2}$ and a sheet resistance of $R_{f}=50 \Omega / \mathrm{sq}$ (this is meant to represent an actual sheet resistance twice as high, but moderated by the fact that the separation between front metal fingers is usually $1-2 \mathrm{~mm}$ ). At the rear surface, we consider the presence, or not, of a diffusion with $R_{b}=100 \Omega / \mathrm{sq}$ and a low recombination factor $J_{O \text { pass }}=30 \mathrm{fA} \cdot \mathrm{cm}^{-2}$, achievable with well-passivated boron and phosphorus diffusions having such sheet resistance. At the location of the metal contact, a $100 \Omega / \mathrm{sq}$ diffusion typically presents a recombination factor of $J_{\text {Ocont }}=1000 \mathrm{fA} \cdot \mathrm{cm}^{-2}$. To make the comparison clearer for the case of a PRC cell with localised diffusions underneath the rear contacts, we retain the same $J_{O \text { cont }}$ and give a very high value to $R_{b}$. It is worth mentioning that a localised $\mathrm{p}^{+}$or $\mathrm{n}^{+}$diffusion could be deeper, with a higher dopant dose, and achieve a $J_{0 \text { cont }}=300 \mathrm{fA} \cdot \mathrm{cm}^{-2}$ even when contacted by metal.

Figures 5 and 6 present the results of the analysis for the case of line contacts of width $d=150 \mu \mathrm{m}$, comparing them with Sentaurus simulations [2]. The results for p-type locally diffused PRC cells were presented in a previous

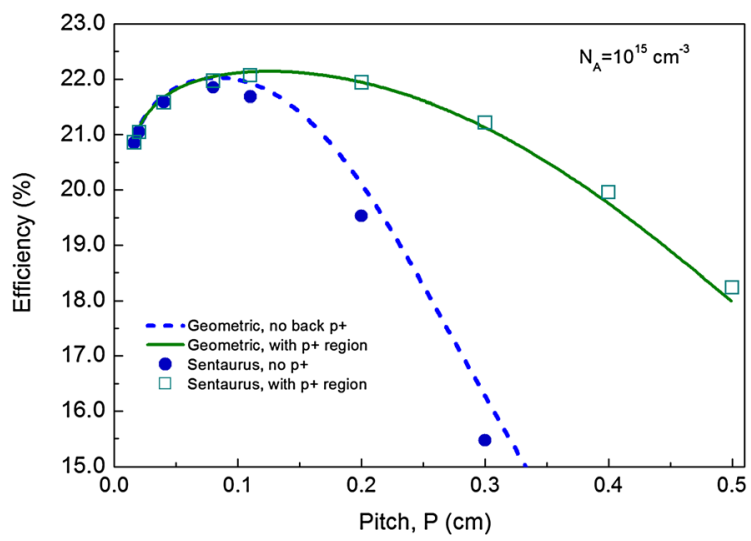

Figure 5. Conversion efficiency as a function of the pitch for a p-type partial rear contact cell with $N_{A}=10^{15} \mathrm{~cm}^{-3}, W=150 \mu \mathrm{m}$ and linear contacts, $d=150 \mu \mathrm{m}$. The front and back surface recombination factors are $J_{\text {of }}=100 \mathrm{fAcm}^{-2}$ and $J_{\text {ocont }}=1000 \mathrm{fAcm}^{-2}$. The case of a full-area $100 \mathrm{Ohm} / \mathrm{sq}$ rear diffusion is shown, with $J_{\text {Opass }}=30 \mathrm{fAcm}^{-2}$ and $J_{\text {Ocont }}=1000 \mathrm{fAcm}^{-2}$.

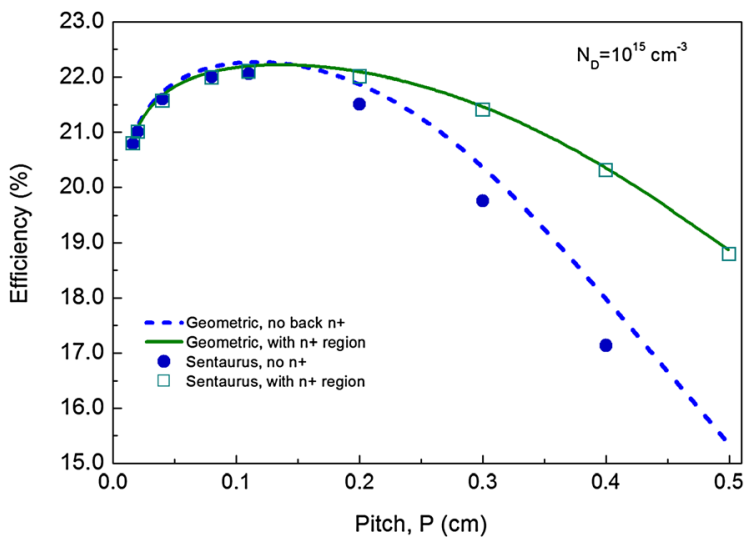

Figure 6. Conversion efficiency as a function of the pitch for an n-type partial rear contact cell with $N_{D}=10^{15} \mathrm{~cm}^{-3}$. Same parameters as in Figure 5. 
article [13]; an incorrect setting of bandgap narrowing parameters in Sentaurus led to lower $V_{\mathrm{oc}}$ values than the geometric model. This has now been resolved, and we have found a good agreement between Sentaurus and the geometric model for all the electrical parameters of the solar cell [20]. Such agreement can be observed in Figures 5 and 6 in terms of the conversion efficiency. The agreement is also excellent for the case of devices with a full rear diffusion, which validates the simple approach described in Section 3. The results indicate that the addition of such layer does not increase the maximum efficiency, but it makes the optimisation of the pitch less critical. The impact of the back diffusion is more significant in the case of a $13.5 \Omega \mathrm{cm}$ p-type wafer than in the case of a $4.8 \Omega \mathrm{cm}$ n-type wafer. The maximum efficiency is practically the same for both types, due to the fact that contact recombination, represented by $J_{O \text { cont }}=1000 \mathrm{fA} \cdot \mathrm{cm}^{-2}$, is relatively moderate. To understand the physics, it is useful to go back to Figure 4, where the carrier concentration profiles in the lateral region are given for the p-type solar cell and for $P=0.4 \mathrm{~cm}$. In maximum power, the addition of a full rear diffusion leads to a drastic reduction of the carrier concentration and therefore to much lower recombination losses in the peripheral region; this results in a higher output current and power.

\section{2. n-type versus p-type silicon partial rear contact solar cells}

Let us explore further the question of whether n-Si can be advantageous compared with $\mathrm{p}-\mathrm{Si}$ to make PRC solar cells. We establish the comparison at an equal dopant density of $N_{\text {dop }}=10^{16} \mathrm{~cm}^{-3}$, because bulk and surface recombination and device voltage are determined by the product of the majority and minority carrier concentrations. The main difference between the $1.45 \Omega \mathrm{cm}$ p-type and the $0.53 \Omega \mathrm{cm}$ n-type wafers is that the mobilities of the majority and minority carriers are swapped, and this can have significant repercussions in a device like the PRC whose essence is the control of the effective conductance for minority charge carriers. Figures 7 and 8 compare the case of n-Si and $\mathrm{p}-\mathrm{Si}$ in terms of $V_{\mathrm{oc}}$ and efficiency for a range of pitch values, while maintaining a fixed point-contact diameter $d=100 \mu \mathrm{m}$ and a front surface recombination parameter $J_{\text {Of }}=10^{-13} \mathrm{Acm}^{-2}$. Two different rear contacts are considered, the first presenting a very high recombination velocity, approximately equal to the kinetic limit $S_{\text {cont }}=3 \times 10^{6} \mathrm{~cm} \cdot \mathrm{s}^{-1}$, that is, $J_{O \text { cont }}=5 \mathrm{nAcm}^{-2}=$ $5 \times 10^{-9} \mathrm{Acm}^{-2}$; the second contact is well passivated, with $J_{O \mathrm{cont}}=300 \mathrm{fAcm}^{-2}=3 \times 10^{-13} \mathrm{Acm}^{-2}$, representative of the lowest value achievable with a metalcontacted boron or phosphorus diffusion. For the Ohmic contact case, n-Si offers a clear advantage, but a wellpassivated contact makes the difference in carrier mobilities almost irrelevant; the n-type device is more tolerant to large pitch values, thanks to its better lateral conductivity for majority carriers. It is also interesting to note that $V_{\mathrm{oc}}$ saturates to a lower value for the case

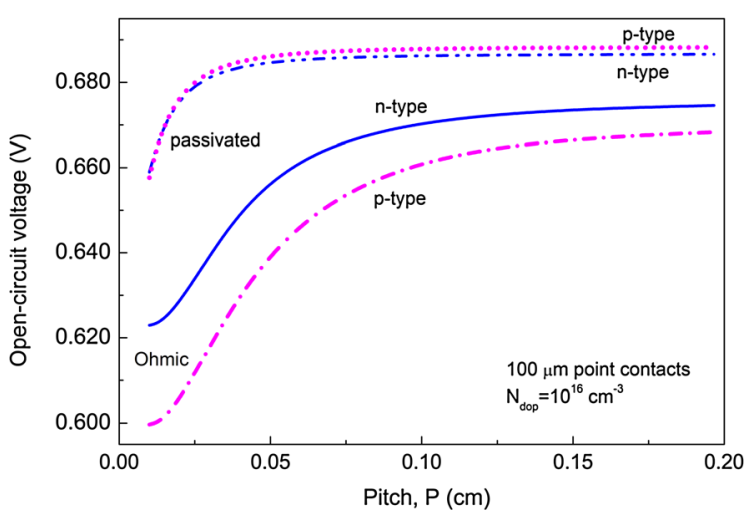

Figure 7. Open-circuit voltage as a function of the pitch for point contacts of diameter $d=100 \mu \mathrm{m}$ and $J_{\text {ocont }}=5 \mathrm{nAcm}^{-2}$ (Ohmic) or $J_{\text {Ocont }}=300 \mathrm{fAcm}^{-2}$ (passivated). Other parameters are $N_{\text {dop }}=10^{16} \mathrm{~cm}^{-3}, W=150 \mu \mathrm{m}$ and $J_{\text {Of }}=100 \mathrm{fAcm}^{-2}$.

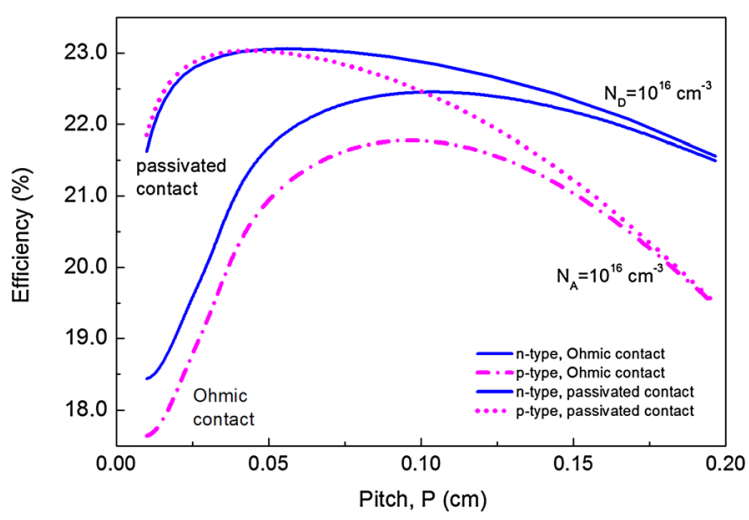

Figure 8. Efficiency as a function of the pitch for point contacts of diameter $d=100 \mu \mathrm{m}$ and $J_{\text {Ocont }}=5 \mathrm{nAcm}^{-2}$ (Ohmic) or $J_{\text {Ocont }}=$ $300 \mathrm{fAcm}^{-2}$ (passivated). Other parameters are $N_{\text {dop }}=10^{16} \mathrm{~cm}^{-3}$, $W=150 \mu \mathrm{m}$ and $J_{\text {of }}=100 \mathrm{fAcm}^{-2}$.

of high contact recombination and high minority carrier mobility (i.e. for p-type Si). The reasons for such saturation are explained in [13].

\subsection{Points versus lines and the impact of contact resistance}

Although practical reasons may eventually determine the decision, it is interesting to discuss what advantages in performance may result from localised circular contacts compared with linear rear contacts. Figure 9 presents a comparison between both for the case of a contact recombination rate equal to the kinetic limit, represented by $J_{O \text { cont }}=5 \mathrm{nAcm}^{-2}$ and an n-type substrate with $N_{D}=10^{16} \mathrm{~cm}^{-3}$. The optimum pitch values are 0.1 or $0.24 \mathrm{~cm}$ for circular or linear contacts, respectively. In the absence of any contact resistance, point contacts are advantageous over line contacts of the same dimension $(100 \mu \mathrm{m}$ has been used for this comparison), providing an efficiency gain of $0.6 \%$ absolute. It seems surprising that line contacts 


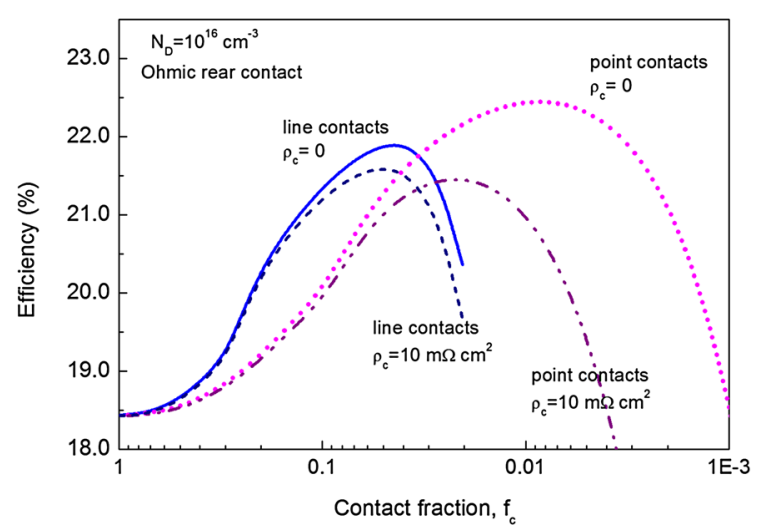

Figure 9. Efficiency as a function of the metal contact fraction for $d=100 \mu \mathrm{m}$ point contacts or $d=100 \mu \mathrm{m}$ line contacts. The n-type cell, $N_{D}=10^{16} \mathrm{~cm}^{-3}$. Contact resistance either 0 or $\rho_{c}=10 \mathrm{~m} \Omega \mathrm{cm}^{2}$. Other parameters are $W=150 \mu \mathrm{m}$, $J_{\text {Ocont }}=5 \times 10^{-9} \mathrm{Acm}^{-2}$ and $J_{\text {Of }}=100 \mathrm{fAcm}^{-2}$.

cannot provide the same level of efficiency as point contacts. The reason is that to achieve a given level of contact fraction, lines need to be spaced much further apart than points, which exacerbates the losses associated with the lateral transport of carriers.

In all the examples presented until now the contact resistance between the metal and the semiconductor has been neglected. This is usually a reasonable assumption if a dopant diffusion is present underneath the contact, but it can be problematic for PRC cells where the wafer substrate is directly contacted by a metal. The comparison between point and line rear contacts, but adding now the impact of a contact resistivity of $\rho_{c}=10 \mathrm{~m} \Omega \mathrm{cm}^{2}$, is repeated in Figure 9 . Such impact is much more severe in the case of point contacts than for linear contacts. The latter do not suffer much from contact resistivity because the optimum pitch occurs at a relatively large contact fraction of $4 \%$, for which contact resistance losses are small. In fact, the optimum does not shift significantly, compared with the case of zero contact resistivity. In the case of point contacts, the optimum contact fraction is slightly less than $1 \%$ if contact resistance is negligible, but it needs to be increased to about $2 \%$ if $\rho_{c}=10 \mathrm{~m} \Omega \mathrm{cm}^{2}$. Even so, contact resistance still causes a drop of $16 \mathrm{mV}$ in the maximum power voltage and a drop in efficiency of $1 \%$ absolute, bringing its value to approximately the same level (slightly lower) achieved with linear contacts. Hence when contact resistivity is high, point contacts do not bring a significant advantage over linear contacts.

\subsection{Impact of bulk recombination}

As expressed in (18), a key simplification to calculate recombination losses in the peripheral region has been to assume an approximately constant carrier concentration from the front to the back. Such assumption can be compromised in the presence of defect-assisted recombination in the bulk of the wafer. The simplification is particularly drastic in short-circuit conditions, because it forces a zero carrier concentration $p_{f}$ for lateral distances in the vicinity of $y_{\min }$, and therefore underestimates bulk recombination. In reality, the carrier concentration in the vertical direction is always greater than zero, following a specific shape that depends on the generation and recombination rates as a function of depth. Such shape does not vary greatly in short-circuit conditions for the type of solar cells we are concerned with in this paper, that is, with a well-passivated rear surface (we are considering now the peripheral region) and a minority carrier diffusion length that is greater than the thickness of the wafer. In fact, it is possible to evaluate the carrier profile based only on the generation rate as a function of position, which can be determined with a simple optical calculation. For the case of a $150 \mu \mathrm{m}$-thick p-type wafer with doping $N_{A}=10^{16} \mathrm{~cm}^{-3}$, and a photogenerated current density $J_{\mathrm{ph}}=40 \mathrm{~mA} \cdot \mathrm{cm}^{-2}$ created by the AM1.5G solar spectrum, the average carrier concentration in short-circuit is $n_{\mathrm{av}(\mathrm{sc})}=1.1 \times 10^{13} \mathrm{~cm}^{-3}$. The determination of recombination losses in the peripheral region can be improved by adding to (18) the extra bulk recombination term associated to $n_{\mathrm{av}(\mathrm{sc})}$. More details on the determination of $n_{\mathrm{av}(\mathrm{sc})}$ are given in a companion conference paper [20].

To test the method described previously, let us consider the boron-oxygen (BO) defect in p-type Czochralski silicon with oxygen and boron concentrations $\left[O_{i}\right]=7.5 \times 10^{17} \mathrm{~cm}^{-3}$ and $N_{A}=10^{16} \mathrm{~cm}^{-3}$. The minority carrier diffusion lengths for the degraded and cured states are 290 and $810 \mu \mathrm{m}$, according to the parameterizations proposed by Bothe et al. [21] and by Schmidt et al. [22], respectively. We select a front surface region with $J_{O f}=100 \mathrm{fA} \cdot \mathrm{cm}^{-2}$ and $R_{f}=50 \Omega / \mathrm{sq}$, a perfectly passivated rear surface, and localised $100 \mu \mathrm{m}$ line contacts with $J_{O \text { cont }}=1000 \mathrm{fA} \cdot \mathrm{cm}^{-2}$. Figure 10 shows the conversion efficiency as a function of the pitch for the cases of intrinsic bulk recombination, the inactivated BO defect (cured lifetime) and the activated BO defect (degraded lifetime).

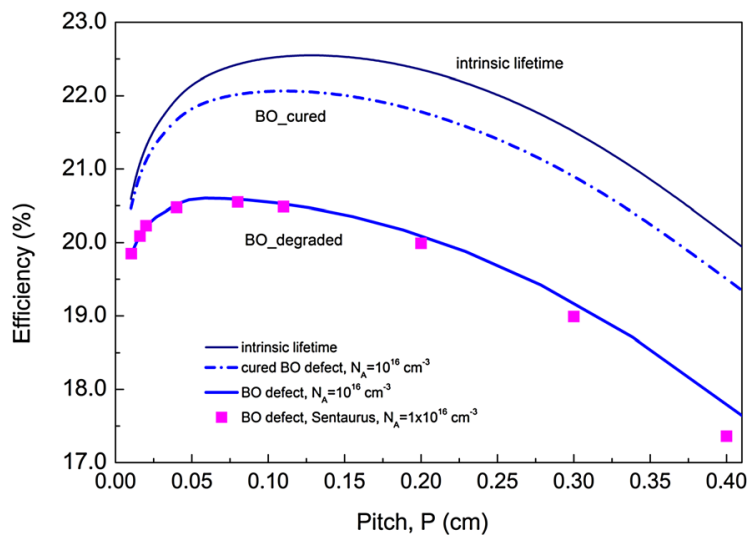

Figure 10. Efficiency as a function of the pitch for $d=100 \mu \mathrm{m}$ line contacts, $p$-type silicon with a boron concentration of $1 \times 10^{16} \mathrm{~cm}^{-3}$ in the intrinsic limit or with boron-oxygen defects due to an oxygen concentration of $7.5 \times 10^{17} \mathrm{~cm}^{-3}$, either in the cured or degraded state. 
The latter case has also been modelled with Sentaurus device. The agreement between the geometric model and Sentaurus is quite good over a broad range of pitch values. The maximum efficiency drops from $22.5 \%$ for intrinsic recombination to $22.1 \%$ for the case of the cured $\mathrm{BO}$ defect and down to $20.6 \%$ for the degraded BO defect. The optimum pitch shifts slightly towards smaller values, from 1.3 to 1 and $0.6 \mathrm{~mm}$, respectively, but the maxima are quite broad.

Note that the results in Figure 10 are not directly comparable with those presented in [22], because the surface recombination parameters are not identical and we have not accounted for shading losses due to the front metal grid. It is worth noting that the efficiency for the $1 \mathrm{D}$ case with a full-area rear metal contact $(P=0)$ has been slightly overestimated throughout this paper, due to the fact that we have kept the same optical properties, that is, the same $J_{\mathrm{ph}}$, independently of the fraction of the rear surface covered by the contacts. A good discussion of such dependence can be found in [23].

\section{CONCLUSION}

By partitioning the PRC solar cell into a central region near the contact and a peripheral region around it, it is possible to visualise the $2 \mathrm{D} / 3 \mathrm{D}$ transport of electrons and holes, their generation and their recombination. The peripheral region serves to absorb photons and transfer electrons and holes towards the central region, but not before many recombine in-situ. That recombination is accentuated by the fact that the periphery operates at a higher internal voltage, which can reach open-circuit conditions in extreme situations. The low fill factor of PRC cells that have a long separation between contacts is largely due to recombination in the peripheral region and to a reduced contribution from it to the output current. We have seen that adding a continuous dopant diffusion on the rear surface facilitates the lateral transport of carriers and makes it possible for the peripheral region to be larger; but this does not necessarily lead to a higher conversion efficiency; it just means that it is possible to use a larger pitch between contacts.

Globally, the PRC solar cell design is a clever implementation of the idea that the minority carrier conductance can be geometrically manipulated to curtail recombination losses at the rear surface. The constriction of the flow of carriers towards a small contact area is equivalent to a reduction of the conductance (or an increase of the resistance), for both electrons and holes, in the direction of the rear contact. By using n-type instead of p-type silicon wafers, the low hole mobility and the high electron mobility can be used to advantage. The n-type $\mathrm{Si}$ is beneficial to reduce minority carrier recombination at the rear contact when it is very high and to facilitate majority carrier transport in the lateral region. Nevertheless, when contact recombination is low, p-type $\mathrm{Si}$ gives approximately the same level of performance, even if the optimization of the pitch is somewhat more critical. The $\mathrm{p}$-Si has a higher intrinsic lifetime than $\mathrm{n}-\mathrm{Si}$ at moderate and high dopant densities [24]; this makes it preferable if recombination at the front diffusion can be suppressed. On the other hand, $\mathrm{p}-\mathrm{Si}$ is more prone to defect-assisted recombination.

Both a high bulk recombination and a high wafer resistivity tend to shift the optimum pitch towards smaller values, but in general such optimum is not critical. The applicability of the model presented here is limited to cases where the minority carrier diffusion length is considerably higher (at least three times) than the wafer thickness. We have confirmed that it is sufficiently accurate to indicate the main trends related to the common BO defect encountered in Czochralski silicon.

Contact resistivity has a stronger impact on pointcontact than on line-contact devices. The reason is that the latter require a higher contact fraction for maximum performance. On the other hand, if contact resistivity is very low, point contacts lead to higher conversion efficiencies. This is because they can be more closely spaced, thus avoiding much of the loss due to lateral carrier transport in the peripheral region. Smaller contact sizes lead to some improvement in cell efficiency but rather small. With point contacts, adding a full-area diffusion to the rear surface brings practically no benefit in performance, although it permits to place them further apart.

To some extent, 2D carrier transport effects are present in all silicon solar cells. The lateral transport of current along the front-diffused region produces an internal voltage that increases with distance from the metal fingers, although not by much if the front metal grid is well designed. In addition, the thermal recombination current density $J_{O f}$ is much higher in the parts of the front diffusion that are contacted by metal than in those that are passivated. In this paper, we have assumed a single value of $J_{O f}$ everywhere, but the regional analysis used for the local rear contacts could be extended to the front side. This and other refinements to the geometric model would be interesting topics to discuss on another occasion.

\section{ACKNOWLEDGEMENT}

The author is indebted to Di Yan of the Australian National University (ANU) for the Sentaurus device simulations presented in this paper.

\section{REFERENCES}

1. Sinton RA, Kwark Y, Gan JY, Swanson RM. 27.5percent silicon concentrator solar cells. IEEE Electron Devices Letters 1986; EDL-7: 567-569.

2. Sentaurus Process User Guide, Version E-2010-12. Synopsys, Inc.: Mountain View, CA, 2010.

3. Basore PA, Cabanas-Holmen K. PC2D: a circularreference spreadsheet solar cell device simulator. IEEE Journal of Photovoltaics 2011; 1: 72-77. 
4. Brendel R. Modeling solar cells with the dopantdiffused layers treated as conductive boundaries. Progress in Photovoltaics: Research and Applications 2012; 20: 31-43.

5. Fell A. A free and fast three-dimensional/twodimensional solar cell simulator featuring conductive boundary and quasi-neutrality approximations. IEEE Transactions on Electron Devices 2013; 60: 733-738.

6. Fisher B. Loss analysis of crystalline silicon solar cells using photoconductance and quantum efficiency measurements. PhD Thesis, University of Konstanz, 2003.

7. Plagwitz H, Brendel R. Analytical model for the diode saturation current of point-contacted solar cells. Progress in Photovoltaics: Research and Applications 2006; 14: 1-12.

8. Saint-Cast P, Rudiger M, Wolf A, Hofmann M, Rentsch J, Preu R. Advanced analytical model for the effective recombination velocity of locally contacted surfaces. Journal of Applied Physics 2010; 108: 013705-013705-013707.

9. Kimmerle A, Rüdiger M, Wolf A, Hermle M, Biro D. Validation of analytical modelling of locally contacted solar cells by numerical simulations. Energy Procedia 2012; 27: 219-226.

10. Wolf A, Biro D, Nekarda J, Stumpp S, Kimmerle A, Mack S, Preu R. Comprehensive analytical model for locally contacted rear surface passivated solar cells. Journal of Applied Physics 2010; 108: 124510.

11. Cuevas A. Geometrical analysis of solar cells with partial rear contacts. IEEE Journal of Photovoltaics 2012; 2: 485-493.

12. Cuevas A, Petermann JH. Geometric analysis of solar cells with partial rear contacts: comparison to 3-D simulations. IEEE Journal of Photovoltaics 2013; 3: $587-589$.

13. Cuevas A. Physical model of back line-contact frontjunction solar cells. Journal of Applied Physics 2013; 113: 164502.

14. Cuevas A, Yan D, Haasse F, Petermann J, Brendel R. A comparison of models to optimize the local contact of partial rear contact solar cells. Energy Procedia 2013; 38: 13-21.

15. Würfel P. Physics of Solar Cells: from Basic Principles to Advanced Concepts. Wiley-VCH: Berlin, 2009; ISBN 978-3-527-40857-3.

16. Swirhun SE, Swanson RM. Temperature dependence of specific contact resistivity. Electron Device Letters, IEEE 1986; 7: 155-157.

17. Swirhun SE. Characterization of majority and minority carrier transport in heavily doped silicon. PhD thesis, Stanford University 1987.

18. Cuevas A, Sinton RA. Detailed modelling of silicon solar cells. In Proceedings 23rd European Photovoltaic Solar Energy Conference, WIP-Renewable Energies, Lincot D, Ossenbrink H, Helm P (eds). Valencia, Spain, 2008; 315-319.

19. Hargreaves S, Black LE, Yan D, Cuevas A. Modelling silicon solar cells with up-to-date material parameters. Energy Procedia 2013; 38: 66-71.

20. Cuevas A. Electrons and holes in solar cells with Partial Rear Contacts. In Proceedings 28th European Photovoltaic Solar Energy Conference. WIP-Renewable Energies: Paris, 2013.

21. Bothe K, Sinton R, Schmidt J. Fundamental boronoxygen-related carrier lifetime limit in mono- and multicrystalline silicon. Progress in Photovoltaics: Research and Applications 2005; 13: 287-296.

22. Schmidt J, Lim B, Walter D, Bothe K, Gatz S, Dullweber T, Altermatt PP. Impurity-related limitations of next-generation industrial silicon solar cells. IEEE Journal of Photovoltaics 2013; 3: 114-118.

23. Muller J, Gatz S, Bothe K, Brendel R. Optimizing the geometry of local aluminum-alloyed contacts to fully screen-printed silicon solar cells. In 38th IEEE Photovoltaic Specialists Conference (PVSC), 2012; 002223-002228.

24. Richter A, Glunz SW, Werner F, Schmidt J, Cuevas A. Improved quantitative description of Auger recombination in crystalline silicon. Physical Review B 2012; 86: 165202 . 